Article

\title{
Deep but Not Periventricular White Matter Disease Is a Marker for Frailty in Older Patients with Early Alzheimer's Disease
}

\author{
Chin Yee Cheong 1,2,*, Robin Wai Munn Choo ${ }^{2}$, Ngoc Huong Lien Ha ${ }^{2}$, \\ Ivana Wei Xin Chan ${ }^{2}$, Aliza Wong ${ }^{3}$, Lin Wee ${ }^{3}$, Philip Lin Kiat Yap ${ }^{1,2}$ \\ 1 Department of Geriatric Medicine, Khoo Teck Puat Hospital, 90 Yishun Central, \\ S768828, Singapore \\ 2 Geriatric Education and Research Institute, 2 Yishun Central 2, S768024, \\ Singapore \\ 3 Lee Kong Chian School of Medicine, 59 Nanyang Dr, Experimental Medicine \\ Building, S636921, Singapore \\ * Correspondence: Chin Yee Cheong, Email: cheong.chin.yee@ktph.com.sg; \\ Tel.: +65-6555-8000.
}

\begin{abstract}
Background: Frailty and cognitive impairment are closely related in sharing several possible underlying pathophysiological mechanisms. There is a lack of clarity in published literature on whether cerebral infarcts or Alzheimer's pathology accounts for the frailty phenotype in patients with mild Alzheimer's disease (AD). Therefore, we investigated the structural neuroimaging predictors of frailty in mild AD patients to better elucidate the underlying pathophysiology.
\end{abstract}

Methods: We recruited subjects who satisfied inclusion criteria from a clinical database of patients attending a tertiary hospital memory clinic between 2012 and 2017. AD patients with Clinical Dementia Rating (CDR) 0.5 and 1.0 who had undergone MRI brain were included. Frailty Index (FI-CGA) was utilised for frailty assessment, and visual MRI rating scales were used by blinded raters to quantify the brain lesions.

Results: A total of 342 mild AD patients with a mean age of $75.79 \pm 7.18$ were studied. A multivariate linear regression model adjusted for demographics,

\section{G Open Access}

Received: 04 May 2019

Accepted: 13 August 2019

Published: 16 August 2019

Copyright $\odot 2019$ by the author(s). Licensee Hapres, London, United Kingdom. This is an open access article distributed under the terms and conditions of Creative Commons Attribution 4.0 International License. cognitive scores and functional status revealed only deep white matter hyperintensities (DWMH) but none other brain lesions to be significantly and positively correlated with FI-CGA ( $\beta=0.178, \mathrm{SE}=0.047, p \leq 0.001)$ This model which comprised age, CDR sum of boxes, basic activities of living and DWMH, accounted for $47.5 \%$ of the FI-CGA variance in the study population.

Conclusions: The study has revealed DWMH to be independently associated with frailty in mild $\mathrm{AD}$ patients. With the current understanding of the aetiology of DWMH, control of vascular risk factors is vital to preventing and ameliorating frailty in patients with mild AD.

KEYWORDS: deep white matter hyperintensities; frailty; early dementia 


\section{ABBREVIATIONS}

MRI, magnetic resonance imaging; DWMH, deep white matter hyperintensities; FI-CGA (Frailty Index-Comprehensive Geriatric Assessment), Clinical Dementia Rating (CDR)

\section{INTRODUCTION}

Frailty is a state of increased vulnerability due to decreased physiologic reserves and dysregulation of multiple physiologic systems [1]. Although physical impairment is the hallmark of frailty, cognitive impairment has also been found to impact physical frailty with adverse outcomes such as functional disability, hospitalisation, institutionalisation and mortality [2,3]. Other studies have also reported an association between physical frailty and cognitive impairment [4-6] and the mechanisms proposed include vascular disease, subcellular disturbances, systemic disorders, chronic inflammation, and impaired mitochondrial function [6,7].

On Magnetic Resonance Imaging (MRI) of the brain, WMH is a surrogate marker of cerebral small vessel disease while the typical patterns of atrophy of Alzheimer's disease can also be observed on neuroimaging [8,9]. Studies have shown a positive association between frailty as assessed by the frailty index, and severity of white matter hyperintensities (WMH) in general older population but the evidence in patients with Alzheimer's disease $(\mathrm{AD})$ is limited $[10,11]$. On the other hand, Alzheimer pathology but not cerebral infarcts have been reported to be associated with increased levels of frailty. However, as those studies were done through autopsied participants using the subcomponents of frailty as outcomes, there are also limitations in generalising the results for clinical purposes [12-14].

The contradictory findings in extant literature led us to undertake this study to examine the relationship between structural neuroimaging characteristics and the frailty phenotype in patients with mild AD. We hope that a better understanding of the underlying pathophysiology derived from neuroimaging can facilitate targeted interventions to attenuate the progression of frailty in patients in $\mathrm{AD}$.

\section{MATERIALS AND METHODS}

\section{Setting and Participants}

Relevant data from suitable patients attending a memory clinic in a tertiary hospital between 2012 and 2017 was extracted from the clinical database. In this clinic, MRI brain was performed as the standard neuroimaging for all patients undergoing evaluation for dementia unless there were contraindications. Ethics approval and consent waiver were obtained from the National Healthcare Group Domain-Specific Review Board (project code: AH2004-0002, approval date: 01 August 2004). 


\section{Diagnosis and Assessment}

The diagnosis of $\mathrm{AD}$ was made via a consensus of a panel comprising geriatricians, a clinical psychologist, and a dementia nurse clinician based on Diagnostic and Statistical Manual of Mental Disorders-IV criteria [15]. Clinical Dementia Rating (CDR), an instrument used to characterise six domains of cognitive and functional performance applicable to AD was used from which a global as well as a total "Sum of Boxes (SOB)" score was generated. A global CDR score of 0 is normal, $0.5,1,2$, and 3 denote very mild dementia, mild dementia, moderate dementia and severe dementia respectively. A 5-point scale used to characterise the six domains of memory, orientation, judgment and problem solving, community affairs, home and hobbies, and personal care are summed up to derive the SOB score. This score is used as interval data for longitudinal tracking of the severity of dementia [16]. Only patients with very mild or mild AD (CDR of 0.5 to 1.0) were included [17]. We collected baseline demographics and cognitive function with the modified version of the Mini-Mental State Exam (MMSE)[18]. For functional assessments, we used (i) modified Barthel Index (MBI) for basic activities of daily living (BADL) with the score ranging from 0 too 100; and (ii) Lawton's instrumental activities of daily living (IADL) with the score ranging from 0 to 14 . For both instruments, higher scores implied better function [19,20].

The frailty index (FI) based on a standard comprehensive geriatric assessment (CGA) derived from clinical examination (FI-CGA) was used to ascertain frailty. It comprises ten impairment items that are assessed individually as part of CGA and added to a count of co-morbidities. FI-CGA has been widely validated, and the scores are divided into seven levels of frailty with level seven as the most frail [21]. It has also been studied widely and proven to be sensitive and significantly associated with shortand long-term adverse health outcomes [22].

Only mild AD patients who had MRI brain performed were recruited into the study. For patients with more than one MRI brain scan, we used the scan taken closest to the date of frailty assessment. Two neuroimaging visual rating scales were used to assess for structural lesions and atrophy:

\section{Brain Atrophy and Lesion Index (BALI)[23]}

BALI is a validated MRI-based visual rating scale developed to describe brain structural variability in $\mathrm{AD}$, mild cognitive impairment and normal ageing. Seven categories are included in the BALI, which covers various common types of lesions observed in both supratentorial and infratentorial regions. A value between 0 and 3 is assigned to each category based on severity, with a higher score denoting greater severity.

\section{Scheltens scale [24]}

This is a visual rating scale used to assess medial temporal atrophy (MTA). It was rated using coronal spoiled gradient echo (SPGR) images on a scale of 0 (no atrophy) to 4 (severe atrophy). 
The neuroimaging characteristics were rated by two trained raters independently. Both raters were blinded to the demographic data, diagnosis and FI-CGA score.

\section{Statistical Analysis}

Baseline characteristics of the patients were summarised as mean \pm standard deviation (SD) for continuous variables and as frequency and percentage for categorical variables. Univariate and multivariate linear regression were performed to estimate the coefficient (Coef.) and 95\% confidence interval $(95 \% \mathrm{CI})$. We used multiple regression analysis to adjust for variables that potentially influenced the relationship between frailty and structural neuroimaging characteristics. These variables included; age, gender, baseline cognitive status (MMSE and CDR SOB) and functional status (BADL and IADL). Analysis of the data was performed using Stata 15.1.

\section{RESULTS}

A total of 342 patients with mild AD, aged $75.79 \pm 7.18$ and with more females (64.04\%) were included. We found that FI-CGA correlated positively with age and CDR SOB, and negatively with MMSE and functional scores. On simple linear regression, only grey matter lesions ( $\beta=0.227, \mathrm{SE}=0.080, p=0.005)$, periventricular hyperintensities $(\mathrm{PVH})$ $(\beta=0.216, \mathrm{SE}=0.058, p \leq 0.001)$, deep white matter hyperintensities $(\mathrm{DWMH})(\beta=0.259, \mathrm{SE}=0.061, p \leq 0.001)$, global atrophy $(\beta=0.208$, $\mathrm{SE}=0.089, p=0.020)$ and MTA $(\beta=0.125, \mathrm{SE}=0.054, p=0.020)$ correlated positively and significantly with FI-CGA (Table 1$)$.

Table 1. Baseline characteristics of the patients and the results of univariate analysis.

\begin{tabular}{|c|c|c|c|c|c|c|c|}
\hline \multirow{2}{*}{\multicolumn{2}{|c|}{ Characteristics }} & \multirow{3}{*}{$\begin{array}{l}\text { Total (SD/\%) } \\
342\end{array}$} & \multicolumn{5}{|c|}{ Linear Regression } \\
\hline & & & \multirow[t]{2}{*}{ Coef. } & \multirow[t]{2}{*}{ SE } & \multicolumn{2}{|c|}{$95 \% \mathrm{CI}$} & \multirow[t]{2}{*}{$p$-value } \\
\hline & & & & & & & \\
\hline Age & & 75.79 (7.18) & 0.044 & 0.007 & 0.030 & 0.057 & $<0.001$ \\
\hline Gender & Female & $219(64.04 \%)$ & 0.132 & 0.107 & -0.079 & 0.343 & 0.219 \\
\hline \multirow{7}{*}{ Imaging Score } & Grey Matter & $0.28(0.64)$ & 0.227 & 0.080 & 0.070 & 0.384 & 0.005 \\
\hline & PVH & $1.71(0.87)$ & 0.216 & 0.058 & 0.103 & 0.330 & $<0.001$ \\
\hline & DWMH & $1.41(0.83)$ & 0.259 & 0.061 & 0.140 & 0.378 & $<0.001$ \\
\hline & Basal Ganglia & $0.33(0.73)$ & 0.136 & 0.070 & -0.002 & 0.274 & 0.054 \\
\hline & Infratentorial & $0.15(0.48)$ & 0.204 & 0.108 & -0.008 & 0.415 & 0.059 \\
\hline & Global Atrophy & $1.74(0.58)$ & 0.208 & 0.089 & 0.033 & 0.382 & 0.020 \\
\hline & MTA & $1.46(0.95)$ & 0.125 & 0.054 & 0.020 & 0.231 & 0.020 \\
\hline \multirow{2}{*}{ Cognitive Score } & MMSE & $19.51(5.16)$ & -0.053 & 0.010 & -0.072 & -0.035 & $<0.001$ \\
\hline & CDR SOB & $4.41(1.85)$ & 0.183 & 0.026 & 0.131 & 0.234 & $<0.001$ \\
\hline \multirow{2}{*}{ Functional score } & MBI (BADL) & $96.78(9.27)$ & -0.062 & 0.004 & -0.070 & -0.053 & $<0.001$ \\
\hline & IADL & $9.79(3.14)$ & -0.124 & 0.015 & -0.153 & -0.094 & $<0.001$ \\
\hline
\end{tabular}


On multivariate linear regression with the model adjusted for all demographics, imaging scores, cognitive scores and functional status, only DWMH remained significantly and positively correlated with FI-CGA ( $\beta=$ $0.200, \mathrm{SE}=0.062, p \leq 0.001)$. PVH $(\beta=-0.003, \mathrm{SE}=0.060, p=0.963)$, global atrophy $(\beta=-0.004, \mathrm{SE}=0.072, p=0.956)$, and MTA $(\beta=-0.036, \mathrm{SE}=0.044$, $p=0.421$ ) were not significant (Table 2).

Table 2. Multiple regression analysis adjusted for all factors on FI-CGA $(n=342)$.

\begin{tabular}{|c|c|c|c|c|c|c|}
\hline \multirow{2}{*}{\multicolumn{2}{|c|}{ Characteristics }} & \multicolumn{5}{|c|}{ Adjusted for all factors } \\
\hline & & Coef. & SE & \multicolumn{2}{|c|}{$95 \% \mathrm{CI}$} & $p$-value \\
\hline Age & & 0.022 & 0.006 & 0.011 & 0.033 & $<0.001$ \\
\hline Gender & Female & 0.031 & 0.086 & -0.139 & 0.201 & 0.724 \\
\hline \multirow[t]{7}{*}{ Imaging Score } & Grey Matter & 0.058 & 0.065 & -0.069 & 0.186 & 0.368 \\
\hline & PVH & -0.003 & 0.060 & -0.121 & 0.115 & 0.963 \\
\hline & DWMH & 0.200 & 0.062 & 0.077 & 0.323 & $<0.001$ \\
\hline & Basal Ganglia & -0.062 & 0.060 & -0.180 & 0.056 & 0.305 \\
\hline & Infratentorial & -0.053 & 0.086 & -0.222 & 0.115 & 0.535 \\
\hline & Global Atrophy & -0.004 & 0.072 & -0.146 & 0.138 & 0.956 \\
\hline & MTA & -0.036 & 0.044 & -0.123 & 0.052 & 0.421 \\
\hline \multirow[t]{2}{*}{ Cognitive Score } & MMSE & -0.011 & 0.010 & -0.031 & 0.010 & 0.313 \\
\hline & CDR SOB & 0.064 & 0.031 & 0.003 & 0.125 & 0.041 \\
\hline \multirow[t]{2}{*}{ Functional score } & MBI (BADL) & -0.054 & 0.005 & -0.063 & -0.045 & $<0.001$ \\
\hline & IADL & -0.012 & 0.017 & -0.045 & 0.021 & 0.465 \\
\hline
\end{tabular}

\section{DISCUSSION}

The study findings demonstrate a novel association between DWMH and frailty in mild AD patients after adjusting for age and baseline cognitive as well as physical function. No other neuroimaging characteristic was associated with frailty.

The role of the central nervous system in the pathophysiology of frailty has been a subject of interest in recent years especially with the introduction of the concept of cognitive frailty [25]. WMH is recognised as a manifestation of cerebral small vessel disease with significant clinical and pathological outcomes [26]. WMH has been associated with a decline in executive function, falls, low level of physical activities, and even sarcopenia. Our finding is consistent with earlier studies which showed an association between frailty status and $\mathrm{WMH}[10,11,27,28]$. In the multicenter European LADIS study that spanned over a decade, physical frailty was associated with increasing $\mathrm{WMH}$ severity and continued to have an effect on the rate of transition from independence to disability [29]. However, these studies were not targeted at the AD population and hence had limited direct application to patients with AD. In the Rush Memory and Ageing Project, AD pathology was shown to be associated 
with physical frailty but not cerebral infarcts. However, the study measured physical frailty proximate to death and frailty was assessed with selected components of Fried's definition of frailty as opposed to the full measure of frailty $[12,30]$.

For our study, FI-CGA was administered most proximal time-wise to neuroimaging when the patients first presented to the memory clinic. This could reduce the bias from the potential changes of the FI-CGA status over time. Besides, the study population, in general, had preserved BADL (mean MBI $96.78 \pm 9.27$ ) which reduced floor effect. Both global atrophy and medial temporal lobe atrophy, were not significant in the final model as opposed to previous studies [12,13]. The findings suggest WMH to be the forerunner of frailty in early $\mathrm{AD}$ before the more advanced brain atrophy sets in and aggravates frailty. This has important clinical implications in optimising control of vascular risk factors which can potentially have a beneficial impact on the prevention of frailty among patients with mild AD.

Previous studies on the association between WMH and frailty did not separately evaluate the different subtypes of WMH [10,11,28]. Although the LADIS study group found that deep frontal and PVH were related to falls in older adults, it did not target frail or AD patients [27]. The jury is still out on the dichotomisation of PVH and DWMH, but neuropathological differences between them have been reported which suggest that although cerebral ischaemia is a common aetiology, other mechanisms may be differentially involved. Neuropathological studies show that PVH is characterised by more gliosis, loosening of the white matter fibres and myelin loss. On the other hand, DWMH has less gliosis but with more axonal loss, vacuolization and arteriolosclerosis [31]. Both DWMH and $\mathrm{PVH}$ are separately associated with age but the effect is stronger for PVH [32]. Similarly, hypertension is a risk factor for both but diastolic blood pressure is correlated with only $\mathrm{PVH}$ [33]. Conflicting results in homocysteine association with DWMH and PVH have also been reported [34,35]. Previous clinical studies have found DWMH to be more related to a decline in physical function, gait disturbance, urinary incontinence compared to PVH. In the study by Soriano-Raya JJ et al., despite a relatively young population with mild severity of WMH, DWMH was associated with a three- to four-fold increased risk of impaired psychomotor speed, which is one of the key components of frailty [36]. These results could be explained by histological microinfarcts in DWMH with greater declines in motor abilities and extrapyramidal tract symptoms compared to $\mathrm{PVH}$ [36,37], and lend credence to our key study finding that DWMH is a marker of frailty in early-stage AD. Beyond vascular factors, there are other proposed pathogenic mechanisms include aberration in cerebral blood flow autoregulation, venous collagenosis, blood brain barrier disruption and genetic factors [38]. Hence, further studies are warranted on the other possible pathophysiological differences between PVH and DWMH to enable targeted interventions aimed at preventing and delaying the onset of frailty in this group of patients. 
The limitations of our study include its cross-sectional methodology and inability to derive conclusions on causality between DWMH and frailty. However, these findings help to provide the basis for a prospective longitudinal study to further elucidate the causal relationship between DWMH and frailty. Another limitation lies in the use of visual rating scales instead of volumetric MRI measurements which offer more reliable, sensitive and objective results [39]. However, this technology is not easily accessible and used mostly in research settings whereas this study was based on a memory clinic patient population. Finally, the prevalence of the cerebrovascular disease in $\mathrm{AD}$ is known to be higher in the Asia context which could potentially affect the generalisability of the results to other populations [40].

\section{CONCLUSIONS}

Overall, our study has revealed an independent relationship between DWMH and frailty in mild AD. Given the current understanding of the aetiology of DWMH, the biological underpinnings of frailty appear to involve vascular factors beyond mere neurodegeneration. While we await studies to better understand the pathophysiology of DWMH, it would appear for now that control of vascular risk factors is vital to preventing and ameliorating frailty in patients with mild AD. The results also support the notion of analysing WMH separately as it is DWMH and not PVH that has emerged as a marker of frailty in patients with AD. Finally, as frailty can already be found in older patients with early dementia, a comprehensive geriatric assessment is warranted in this group of patients presenting to the memory clinic.

\section{DATA AVAILABILITY}

The dataset from the study is not available due to the institutional data protection policy.

\section{AUTHOR CONTRIBUTIONS}

CYC collected, analysed and interpreted the data, also drafted the manuscript. NHLH, IWXC, AW and LW collected, interpreted the data and revised the manuscript. RWMC analysed part of the data. PLKY conceptualised the study, supervised data collection and analysis, interpreted the data and revised the manuscript.

\section{CONFLICTS OF INTEREST}

The authors declare that there is no conflict of interest. 


\section{REFERENCES}

1. Fried LP, Ferrucci L, Darer J, Williamson JD, Anderson G. Untangling the Concepts of Disability, Frailty, and Comorbidity: Implications for Improved Targeting and Care. J Gerontol Ser A. 2004;59(3):M255-63.

2. Chong E, Ho E, Baldevarona-Llego J, Chan M, Wu L, Tay L. Frailty and Risk of Adverse Outcomes in Hospitalized Older Adults: A Comparison of Different Frailty Measures. J Am Med Dir Assoc. 2017;18(7):638.e7-11.

3. Hubbard RE, Peel NM, Samanta M, Gray LC, Mitnitski A, Rockwood K. Frailty status at admission to hospital predicts multiple adverse outcomes. Age Ageing. 2017;46(5):801-6.

4. Feng L, Nyunt MSZ, Gao Q, Feng L, Lee TS, Tsoi T, et al. Physical Frailty, Cognitive Impairment, and the Risk of Neurocognitive Disorder in the Singapore Longitudinal Ageing Studies. J Gerontol Ser A. 2016;72(3):glw050.

5. Gross AL, Xue Q-L, Bandeen-Roche K, Fried LP, Varadhan R, McAdamsDeMarco MA, et al. Declines and Impairment in Executive Function Predict Onset of Physical Frailty. J Gerontol Ser A. 2016;71(12):1624-30.

6. Robertson DA, Savva GM, Kenny RA. Frailty and cognitive impairment-A review of the evidence and causal mechanisms. Ageing Res Rev. 2013;12: 840-51.

7. Halil M, Cemal Kizilarslanoglu M, Emin Kuyumcu M, Yesil Y, Cruz Jentoft AJ. Cognitive aspects of frailty: Mechanisms behind the link between frailty and cognitive impairment. J Nutr Health Aging. 2015;19(3):276-83.

8. Chabriat H, Levy C, Taillia H, Iba-Zizen MT, Vahedi K, Joutel A, et al. Patterns of MRI lesions in CADASIL. Neurology. 1998;51(2):452-7.

9. Wattjes MP. Structural MRI. Int Psychogeriatrics. 2011;23(S2):S13-24.

10. Avila-Funes JA, Pelletier A, Meillon C, Catheline G, Periot O, Treviño-Frenk I, et al. Vascular Cerebral Damage in Frail Older Adults: The AMImage Study. J Gerontol Ser A. 2017;72(7):971-7.

11. Jung H-W, Kim S-W, Yoon S-J, Choi J-Y, Kim K, Kim C-H. Associations Between Frailty, Retinal Microvascular Changes, and Cerebral White Matter Abnormalities in Korean Older Adults. J Am Geriatr Soc. 2014;62(11):2209-10.

12. Buchman AS, Schneider JA, Leurgans S, Bennett DA. Physical frailty in older persons is associated with Alzheimer disease pathology. Neurology. 2008;71(7):499-504.

13. Burns JM, Johnson DK, Watts A, Swerdlow RH, Brooks WM. Reduced Lean Mass in Early Alzheimer Disease and Its Association With Brain Atrophy. Arch Neurol. 2010;67(4):428-33.

14. Panza F, Solfrizzi V, Frisardi V, Maggi S, Sancarlo D, Addante F, et al. Different models of frailty in predementia and dementia syndromes. J Nutr Health Aging. 2011;15(8):711-9.

15. American Psychiatric Association. Diagnostic and statistical manual of mental disorders. 4th ed. Washington (US): American Psychiatric Association; 2000.

16. O’Bryant SE, Waring SC, Cullum CM, Hall J, Lacritz L, Massman PJ, et al. Staging Dementia Using Clinical Dementia Rating Scale Sum of Boxes Scores. Arch Neurol. 2008;65(8):1091. 
17. Morris JC. The Clinical Dementia Rating (CDR): current version and scoring rules. Neurology. 1993;43(11):2412-4.

18. Feng L, Sian Chong M, Shiong Lim W, Ng TP. The modified Mini-Mental State Examination Test: Normative data for Singapore Chinese older adults and its performance in detecting early cognitive impairment. Singap Med J. 2014;53(7):458-62

19. Shah S, Vanclay F, Cooper B. Improving the sensitivity of the Barthel Index for stroke rehabilitation. J Clin Epidemiol. 1989;42(8):703-9.

20. Lawton MP, Brody EM. Assessment of Older People: Self-Maintaining and Instrumental Activities of Daily Living. Gerontologist. 1969;9(3 Part 1):179-86.

21. Jones D, Song X, Mitnitski A, Rockwood K. Evaluation of a frailty index based on a comprehensive geriatric assessment in a population based study of elderly Canadians. Aging Clin Exp Res. 2005;17(6):465-71.

22. Pilotto A, Rengo F, Marchionni N, Sancarlo D, Fontana A, Panza F, et al. Comparing the Prognostic Accuracy for All-Cause Mortality of Frailty Instruments: A Multicentre 1-Year Follow-Up in Hospitalized Older Patients. PLoS One. 2012;7(1):e29090.

23. Chen W, Song X, Zhang Y, Darvesh S, Zhang N, D’Arcy RCN, et al. An MRI-based semiquantitative index for the evaluation of brain atrophy and lesions in Alzheimer's disease, mild cognitive impairment and normal aging. Dement Geriatr Cogn Disord. 2010;30(2):121-30.

24. Scheltens P, Launer LJ, Barkhof F, Weinstein HC, van Gool WA. Visual assessment of medial temporal lobe atrophy on magnetic resonance imaging: interobserver reliability. J Neurol. 1995;242(9):557-60.

25. Kelaiditi E, Cesari M, Canevelli M, Abellan van Kan G, Ousset P-J, GilletteGuyonnet S, et al. Cognitive frailty: Rational and definition from an (I.A.N.A./I.A.G.G.) International Consensus Group. J Nutr Health Aging. 2013;17(9):726-34.

26. Pantoni L. Cerebral small vessel disease: from pathogenesis and clinical characteristics to therapeutic challenges. Lancet Neurol. 2010;9(7):689-701.

27. Blahak C, Baezner H, Pantoni L, Poggesi A, Chabriat H, Erkinjuntti T, et al. Deep frontal and periventricular age related white matter changes but not basal ganglia and infratentorial hyperintensities are associated with falls: cross sectional results from the LADIS study. J Neurol Neurosurg Psychiatry. 2009;80:608-13. doi: 10.1136/jnnp.2008.154633

28. Kohara K, Okada Y, Ochi M, Ohara M, Nagai T, Tabara Y, et al. Muscle mass decline, arterial stiffness, white matter hyperintensity, and cognitive impairment: Japan Shimanami Health Promoting Program study. J Cachexia Sarcopenia Muscle. 2017;8(4):557-66. doi: 10.1002/jcsm.12195

29. The LADIS Study Group; Poggesi A, Pantoni L, Inzitari D, Fazekas F, Ferro J, et al. 2001-2011: A Decade of the LADIS (Leukoaraiosis And DISability) Study: What Have We Learned about White Matter Changes and Small-Vessel Disease? Cerebrovasc Dis. 2011;32:577-88.

30. Fried LP, Tangen CM, Walston J, Newman AB, Hirsch C, Gottdiener J, et al. Frailty in Older Adults: Evidence for a Phenotype. J Gerontol Ser A. 2001;56(3):M146-57. 
31. Wharton SB, Simpson JE, Brayne C, Ince PG. Age-Associated White Matter Lesions: The MRC Cognitive Function and Ageing Study. Brain Pathol. 2015;25(1):35-43.

32. Nyquist PA, Bilgel M, Gottesman R, Yanek LR, Moy TF, Becker LC, et al. Age differences in periventricular and deep white matter lesions. Neurobiol Aging. 2015;36(4):1653-8.

33. Wen W, Sachdev PS. Extent and Distribution of White Matter Hyperintensities in Stroke Patients. Stroke. 2004;35(12):2813-9.

34. Gao Y, Wei S, Song B, Qin J, Fang H, Ji Y, et al. Homocysteine Level Is Associated with White Matter Hyperintensity Locations in Patients with Acute Ischemic Stroke. PLoS One. 2015;10(12):e0144431.

35. Sachdev P, Parslow R, Salonikas C, Lux O, Wen W, Kumar R, et al. Homocysteine and the Brain in Midadult Life. Arch Neurol. 2004;61(9):1369.

36. Soriano-Raya JJ, Miralbell J, López-Cancio E, Bargalló N, Arenillas JF, Barrios $\mathrm{M}$, et al. Deep versus Periventricular White Matter Lesions and Cognitive Function in a Community Sample of Middle-Aged Participants. J Int Neuropsychol Soc. 2012;18(05):874-85.

37. Park KH, Lee J-Y, Na DL, Kim SY, Cheong H-K, Moon SY, et al. Different Associations of Periventricular and Deep White Matter Lesions with Cognition, Neuropsychiatric Symptoms, and Daily Activities in Dementia. J Geriatr Psychiatry Neurol. 2011;24(2):84-90.

38. Lin J, Wang D, Lan L, Fan Y. Multiple Factors Involved in the Pathogenesis of White Matter Lesions. BioMed Res Int. 2017;2017:9372050. doi: $10.1155 / 2017 / 9372050$

39. van den Heuvel DMJ, ten Dam VH, de Craen AJM, Admiraal-Behloul F, van Es ACGM, Palm WM, et al. Measuring longitudinal white matter changes: comparison of a visual rating scale with a volumetric measurement. Am J Neuroradiol. 2006;27(4):875-8.

40. Chen C, Homma A, Mok VCT, Krishnamoorthy E, Alladi S, Meguro K, et al. Alzheimer's disease with cerebrovascular disease: current status in the AsiaPacific region. J Intern Med. 2016;280(4):359-74.

How to cite this article:

Cheong CY, Choo RWM, Ha NHL, Chan IWX, Wong A, Wee L, et al. Deep but Not Periventricular White Matter Disease Is a Marker for Frailty in Older Patients with Early Alzheimer's Disease. Adv Geriatr Med Res. 2019;1:e190009. https://doi.org/10.20900/agmr20190009 